\title{
Avatar-based Counselling for Psychological Distress in Secondary School Pupils: Pilot
}

\section{Evaluation}

\author{
Mick Cooper \\ University of Roehampton
}

Biljana van Rijn

Evi Chryssafidou

Metanoia Institute

\section{Author note:}

Mick Cooper, Professor of Counselling Psychology, Department of Psychology, University of Roehampton, Holybourne Avenue, London SW15 4JD, mick.cooper@roehampton.ac.uk; Dr Biljana van Rijn, Faculty Head, Faculty of Applied Research and Clinical Practice, Metanoia Institute, 13 North Common Rd, London, W5 2QB, biljana.vanrijn@metanoia.ac.uk; Dr Evi Chryssafidou, Researcher, Faculty of Applied Research and Clinical Practice, Metanoia Counselling and Psychotherapy Service (MCPS), Metanoia Institute, 13 North Common Rd, London, W5 2QB.

Correspondence concerning this article should be sent to Mick Cooper, Professor of Counselling Psychology, Department of Psychology, University of Roehampton, Holybourne Avenue, London SW15 4JD, mick.cooper@roehampton.ac.uk

Funding: This work was funded by the Small Business Research Initiative. 


\section{Avatar-based Counselling for Psychological Distress in Secondary School Pupils: Pilot Evaluation}

This study is a pilot evaluation of a new avatar-based therapeutic tool, ProReal, with psychologically distressed young people within a school setting. In total, 54 young people, aged 12-18 years old, participated in face-to-face avatar-based counselling. Young people used the software to represent themselves and others, their problems and emotions. The primary outcome measure was the Young Person's CORE (YP-CORE). The avatar-based counselling intervention was feasible to implement and acceptable to clients, with $90 \%$ rating the help that they received as good, and less than $20 \%$ dropout. The intervention was associated with small to medium reductions in psychological distress, psychological difficulties and conduct problems. These improvements, however, were significant for male clients only. Outcomes were greater for counsellors who spent more time in training and using the ProReal software.

Keywords. Computer-assisted therapy, Avatar-based therapy, Adolescents, Treatment outcome 
In the emerging field of e-mental health, avatars are increasingly used as an adjunct to psychological therapy in a variety of psychotherapeutic applications and formats (Clough \& Casey, 2011; Kendzor and Hebert, 2017; Rehm et al. 2016). Avatars are virtual representations of self and other people that enable interaction and communication between the represented characters. Avatars have been used to facilitate, or augment, face-to-face therapy (Rehm et al. 2016), as well as other e-mental health formats. These include internetbased distance counselling (cybertherapy) taking place in virtual environments (Anthony \& Nagel, 2014; Witt et al. 2016; Yuen et al. 2013), and psychoeducational interventions guided by automated virtual counsellors (Gaggioli et al. 2003; Provoost et al. 2017;) or motivated by game scenarios and features (Fleming et al. 2012).

Rehm et al. (2013) have identified two forms of 'avatar-assisted face-to-face therapies' (p.2): a 'client as avatar' type and an 'augmented interaction' type. In the former type, the client needs to be represented as an avatar, to take part in the therapy, along with representations of the client's external reality or internal self. The therapist is not necessary represented as avatar; her role is mainly to help with navigating through and reflecting on the represented scenes and client's internal processes. In the second type, the 'augmented interaction' type, the important element of avatar therapy is the interaction of the client with avatars that function as fear or anxiety-provoking stimuli. The client does not need to be represented in the world, but needs to interact with the avatars that are controlled by the therapist. This category relates to the use of avatars in Virtual Reality Exposure Therapy (VRER), introduced as a component of cognitive behavioural therapy (CBT) (Parsons, 2015). VRET is used as treatment for phobias, PTSD, eating disorders, and anxiety disorders, aiming to modify the patients' responses in a controlled therapeutic setting (Meyerbröker \& Emmelkamp, 2010). There is increasing evidence of clinical effectiveness for VRET 
(Morina et al. 2015), such as reductions in the severity of persistent auditory verbal hallucinations (Craig et al. 2018).

A common rationale for developing and researching avatar-based interventions lies in the expectation that increased immersion in a virtual world will help clients to explore and strengthen their inter- and intra-personal relating (Hoch et al., 2012; Yuen et al., 2013). Avatar-based interventions are also hypothesised to facilitate greater self-disclosure (Joinson, 2001) and improve access to therapy due to participants' familiarity with virtual worlds and avatar-mediated environments (Cuijpers, van Straten, Andersson, \& van Oppen, 2008; Musiat, Goldstone and Tarrier, 2014; Przeworski \& Newman, 2006). Such familiarity may also serve to destigmatise the intervention and, for young people, give a greater sense of control. Clients may also benefit from the 'disinhibition effect': of expressing painful thoughts and feelings through a representative entity (Suler, 2004). The National Collaborating Centre for Mental Health (2014) review of e-therapies and computer-based applications concludes that 'young people want e-therapies to be part of the help they are offered' (p.115).

This study is a pilot evaluation of a new form of avatar-based therapy, ProReal, with young people, following primarily the 'client as avatar' model. ProReal is a digital therapeutic tool in which people can create visual representations of their inner and outer worlds, often poignant ones, and then describe and explore them with others (see illustrative screenshot, Figure 1). Drawing elements from the 'augmented interaction' type also, clients can immerse themselves in the created scenarios or worlds, and control how much to engage with a scene at a time and expose themselves to unwanted thoughts or evocative representations. ProReal has been successfully trialled within a therapeutic prison setting (van Rijn, Cooper, Jackson, \& Wild, 2017)and as part of mentalisation-based treatment 
(MBT) for borderline personality disorder (Falconer et al. 2017). This study tests its acceptability, and preliminary outcomes, within a school setting with young people.

\section{Method}

\section{Participants}

Inclusion criteria for school pupils were as follows: aged 12-18, motivated to attend counselling, capable of consenting to participate in research, and greater than $85 \%$ attendance at school. Individuals were excluded from the study if they were at risk of serious harm to self or other, involved with other child and young people's mental health agencies (outside of the school's established counselling service), or likely to move to another school during the period of the study.

School staff referred 76 young people for counselling, with 56 attending an initial assessment (Figure 2). Of the 20 who did not continue to assessment, five young people excluded themselves because they did not like the digital nature of the intervention. Two more participants were excluded by the counsellor, post-assessment, because they were considered at risk of serious harm. This gave a total of 54 clients allocated to avatar-based counselling, 11 of whom had been previously working with their counsellor.

The 54 participants came from eight different schools. The number of participants per school ranged from 3 to 10, with a mean of 5.4 participants per school and a median of 5 . The average number of sessions attended was $4.7(S D=2.5)$. The mean age of the full sample was 14.2 years old $(S D=1.9)($ Table 1$)$. Twenty-three clients $(42.6 \%)$ were male and $31(57.4 \%)$ were female. The majority of participants identified as British white or British other $(62.9 \%)$. Four of the 54 participants $(7.4 \%)$ indicated that they had a disability.

At baseline, 28 participants (51.9\%) were experiencing clinical levels of psychological distress, as defined by the Young Person's CORE (YP-CORE, Twigg et al., 2016). 


\section{Measures}

The primary outcome measure was the YP-CORE (Twigg et al., 2009; Twigg et al., 2016). This is a self-report measure of psychological distress in young people, and the most commonly used outcome measure in secondary-school-based counselling in the UK (Cooper, 2013). Young people are asked to rate their psychological distress on 10 items using a 5-point scale (0-4), giving a total score between 0 and 40, with higher scores indicating greater levels of distress. The YP-CORE measure has been shown to be acceptable to young people, with good levels of internal consistency (Cronbach's $\alpha=.85$, Twigg et al., 2009), test-retest stability (Pearson's $r=.76$, Twigg et al., 2016), and the capacity to differentiate between means for clinical and non-clinical samples $(19.0[\mathrm{SD}=7.5]$ and $9.4[\mathrm{SD}=7.3])$, respectively, Twigg et al., 2016). In the present study, the YP-CORE was used at pre- and post-therapy assessment points. It was also used at the start of each session of avatar-based counselling, so that there was always a final YP-CORE score.

In addition, we assessed psychological difficulties at pre- and post-therapy using the self-report Strengths and Difficulties Questionnaire (SDQ). This is a widely used brief behavioral screening instrument for children and young people aged 11 to 16 , which can also be used to evaluate the outcomes of specific interventions (Goodman, 2001). The measure consists of 25 items which are grouped into five subscales. Four of these subscales are distress-related: emotional symptoms (SDQ-ES), conduct problems (SDQ-CP), hyperactivity (SDQ-HA) and peer problems (SDQ-PP); and one is strengths-related: pro-social (SDQ-PS). Young people are asked to rate the 25 items according to how they have been feeling over the past six months (at pre-therapy) and past month (at post-therapy). The total difficulties score of the SDQ (SDQ-TD) ranges from 0-40, and is generated by summing the scores on the four distress-related scales. Internal consistency on the SDQ-TD has been found to be acceptable (Cronbach's $\alpha=.82$ and .78, Goodman, Meltzer, \& Bailey, 1998; McArthur, Cooper, \& 
Berdondini, 2013), although concerns have been raised regarding the internal consistency of the individual subscales (e.g., Hagquist, 2007).

To assess mental health problems at pre- and post-therapy, we used items from two subscales of the Revised Child Anxiety and Depression Scale (RCADS): the 6-item generalized anxiety disorder (GAD) subscale and the 10-item major depressive disorder (MDD) subscale (Chorpita, Moffitt \& Gray, 2005). Items are rated on a 4-point Likert-scale from 0 ('never') to 3 ('always'), giving ranges of 0-18 for the GAD subscale, and 0-30 for the MDD subscale. The RCADS has been found to be reliable and valid, with a factor structure that maps onto DSM-5 anxiety disorders and depression.

To assess levels of self-compassion at pre- and post-therapy, we used the 8-item Reassured Self subscale of the Forms of Self-Criticising/Attacking and Self-Reassuring Scale (FSCRS; Gilbert, Clarke, Hempel, Miles, \& Irons, 2004). The questionnaire asks respondents to circle a number from 0 ('not at all like me') to 4 ('extremely like me') indicating how they think and feel when things go wrong for them. This gives a range of total scores of 0-32, with higher scores indicating greater self-reassurance. Analysis with an adult sample indicates that this subscale has good validity and reliability (Baião, Gilbert, McEwan, \& Carvalho, 2015; Castilho, Pinto-Gouveia, \& Duarte, 2015; Gilbert et al., 2004). The measure has not been validated for use with young people. However, for the present sample, acceptability - as assessed by item completion - was high, with just one missing item across the respondents. In addition, we found a very good internal consistency at pre-therapy of .90 .

At post-therapy, we also assessed client satisfaction with treatment using the Experience of Service Questionnaire (ESQ) and its 9-item 'Satisfaction with Care' main factor. This factor has been found to be robust, and sensitive to differences between high and 
low scoring respondents (Brown et al., 2014). Scores on this factor range from 0 to 18, with higher scores indicating greater satisfaction.

\section{Procedure}

The protocol was granted ethical approval by the sponsoring university. Consent was sought from the school headteachers, the parents/carers of the participants, the counsellors, and the participants. The recruitment phase for schools and counsellors preceded the clients' recruitment phase, and lasted for approximately two months. In general, the school headteachers and pastoral care teams welcomed participation in the trial as they believed it would increase the available resources for counselling to their students, and that it would appeal to the young people. The main study took place over eight months (September 2015 to April 2016). Clients were recruited as soon as the school and counsellors committed to the research, and up to two months before the end of the study.

Potential participants were referred by the pastoral care team and/or headteachers to the counsellors. The most common reasons for referral were behaviour issues, self-harm, and bereavement support. The counsellors arranged appointments with the prospective participants and ascertained whether they met the eligibility criteria. If they did, the counsellors then asked the young people to complete all outcomes measures (excluding the ESQ), along with a demographics form (pre-therapy assessment point).

After the completion of the intervention, a member of the research team met with the young person and asked them to complete the post-therapy assessment questionnaires.

The intervention. In this study, ProReal was integrated into school-based humanistic counselling (Cooper et al., 2010), to give avatar-based counselling. School-based humanistic counselling is a nondirective intervention for psychological distress in young people. It is based on competences for humanistic psychological therapies for young people (Hill, Roth, \& 
Cooper, 2013), and has been shown to be effective in randomised controlled trials, with an effect size on the YP-CORE of 0.82 at endpoint against pastoral care as usual (Cooper, 2013).

Avatar-based counselling took place weekly in schools. The sessions lasted for approximately one school period (50-60 minutes). The counsellors were advised to use ProReal in every session, unless the client declined to use it.

During ProReal work, the client, with support from the counsellor, is invited to populate the landscape with avatars and props to help create a visual representation of how they see a situation, such as their relation with friends, parents or stressful events in their life. The landscape consists of a large green field, a river with bridges, a ravine and a castle. Avatars are featureless but the software provides functions for changing their size and colour; and for assigning postures, emotional labels, and text that convey their inner thoughts. The client can also add a range of props, such as a ticking bomb, a clock, a treasure chest; label them, and use them metaphorically. The landscape can be viewed from the client's perspective or that of any other 'individual' represented in the virtual environment.

ProReal is hypothesised to support therapeutic change by helping clients make visible their thoughts, feelings, perceptions, and experience of the world; and by helping them see and appreciate their world from different perspectives. This is from the client's own perspective (egocentric), as well as from other generic or specific people's perspective (exocentric) (Flemming et al. 2015), who may also be represented in the virtual world (see, for example, mentalisation therapy by Falconer et al., 2017). The process of changing perspective is also hypothesised to help with the development of self-compassion and empathy. The expression and communication of experiences could be similar to representations in non-digital formats, such as art therapy and sand tray therapy (Knoetze, 2013; Swan \& Schottelkorb, 2013). Similar to sand tray therapy, the ambiguity of representations can possibly trigger "psychodynamic processes such as projection and 
transference, which in turn, elicit a person's strong emotions” (Barak 2007, p. 310). In addition, ProReal aims to provide a way of experimenting with options for change and offers a medium by which people can communicate their worlds to others (ProReal Ltd, 2015).

During the intervention in the schools, the client and the counsellor sat side by side, facing the screen of the laptop, in a quiet room of the school, where counselling usually took place. The role of the counsellor was to support self-exploration, using guidelines detailed in the ProReal User Manual (ProReal Ltd, 2015). A first session of avatar-based counselling typically began with an invitation to clients to build their 'world' within the virtual landscape. The work would then be participant-led, based on the client's aims and goals, and using the humanistic approach to counselling, as detailed in the ProReal User Manual. The counsellor was expected to actively listen to the client's language, empathically enquire into their visual imagery, and tentatively mirror back their understanding of the metaphors. Counsellors were expected to work flexibly with the software, accepting the way the clients preferred to use the system.

To illustrate the use of ProReal, we present here two scenes from a case study [currently under review for publication]. Richard, is a 14-year-old boy with Autistic Spectrum Disorder (ASD), who had 10 sessions of avatar-based counselling as a participant in the current study. Richard felt that he 'did not fit in', and attributed this to his ASD diagnosis. In the first session with ProReal, Richard chose to build a scene by using a section of wall as a barrier, which he labelled 'cannot get past it' and then put a treasure chest behind the wall barrier, which he labelled 'normal' (Figure 1). He then added a black avatar, which he named 'Fitting In', on the other side of the wall, looking towards the treasure chest, and bearing a stressed posture and crying emoticon. His inner voice, represented with a speech bubble (a feature of ProReal), was saying 'I can see it but cannot achieve it', referring to the treasure chest as a representation of normality. This has been a central scene in the course of 
the therapy, evoking a poignant representation of Richard's problematic experiences. In a later session, he returned to increase the number and size of the treasure chests, explaining that his longing to be normal has increased, after narrating more negative experiences of not fitting in.

Counsellors. Overall, eight counsellors participated in the study. All the counsellors were females, with an average professional experience of 6.5 years. Their training was primarily in humanistic or integrative forms of counselling, with additional training in work with children and young people. Just one of the counsellors had experience of avatar-based work prior to the study.

The counsellors were provided systematic training in the use of ProReal, and were then supported in their delivery of the intervention throughout the period of the study. The training did not focus on the detail of the therapeutic intervention, but on the use of the software within the participant-led therapeutic approach. Additionally, as part of their training for avatar-based counselling, the counsellors were provided with a framework of the ProReal competencies mapped to the competences for humanistic therapy with young people (Hill et al., 2013). Systematic training by ProReal Ltd. was provided both via online and face-to-face methods over a period of two to four weeks. As part of this training, the counsellors were encouraged to practice with other trainees, as well as friends and relatives.

\section{Analysis}

We assessed the feasibility and acceptability of the intervention by considering the amount of time ProReal was used during the sessions, levels of dropout, client satisfaction with the intervention, and the emergence of any major ethical concerns. Clients' and counsellors' experiences of participating in avatar-based counselling were also explored through qualitative interviews, and this is reported separately (van Rijn, Cooper, \& Chryssafidou, 2018).. Pre- and post-therapy scores were compared on all measures to identify 
psychological changes associated with participation in the avatar-based counselling. Effect sizes were calculated using Cohen's $d$, with the standard deviation at pre-therapy serving as the denominator (Stiles et al., 2006).

For the YP-CORE, predictors of outcomes were analysed using t-tests and analysis of variance. Here, the independent variable was the predictor, and the dependent variable was the standardised residual scores of post-therapy outcomes against pre-therapy scores. As our analysis was focused on a novel intervention, we used a cutoff point of $p \leq .1$ for indications of a trend, with $p \leq .05$ for indications of a significant effect. To limit the risks of multiple testing, we restricted our analysis of predictors of other outcomes to those that showed association with the YP-CORE.

Criteria for reliable and clinically significant change, by gender and age group, were taken from Twigg et al. (2016). For reliable change from pre- to post-therapy, YP-CORE scores must change by more than 8.3 points (male, $11-13$ years), 8.0 points (male, $14-16$ years and female, 11-13 years) and 7.4 points (female, 14-16 years). For clinical change, scores must cross the following YP-CORE cutoff points: 10.3 (male, 11-13 years), 14.1 (male, 14-16 years), 14.4 (female, 11-13 years) and 15.9 (female, 14-16 years).

We used multi-level modelling (MLWiN, 2.30) to examine the association between outcomes and the amount of time that each counsellor had spent logged in to ProReal for training or for practice purposes (prior to commencing with actual clients).

Data on the duration (in minutes) of use of the ProReal software were available for 99 sessions from just 24 clients of the clients $(72.8 \%$ of these clients' total sessions of counselling). This was due to technical problems in recoding the sessions through screen capture.

\section{Results}

\section{Preliminary Analysis}


The average amount of ProReal use per session of avatar-based counselling varied from 17.6 to 46.3 minutes, with a mean and median of 33.1 minutes $(S D=8.1)$. Of the 43 new clients (i.e., excluding those who had not worked previously with their counsellor), two (4.7\%) did not attend any avatar-based therapy, and six (14.0\%) dropped out before a planned ending (three after session one, and three after two to eight sessions). Chi-squared tests indicated that female participants had a significantly greater likelihood of dropping out than male participants $\left(25.9 \%\right.$ vs. $4.5 \%$ respectively, $\left.\chi^{2}(1)=4.06, p=.04\right)$.

\section{Psychological distress (YP-CORE)}

At pre-therapy, the mean YP-CORE score for the 52 participants with paired scores was 16.2. At post-therapy it was 12.4 (see Table 2 ). This reduction was significant $(t=3.4$, $p=.001$ ) with an effect size of 0.41: in the small to medium range. For new clients only, there was a significant reduction in psychological distress on the YP-CORE from $16.3(S D=$ $9.5)$ to $11.3(S D=9.8)(t=3.9, p<.001)$, with a moderate effect size of 0.53 . By contrast, pre-existing clients showed no significant change in levels of psychological distress $(t=-0.4$ $p=.71)$

Of the sample who attended avatar-based counselling for one session or more $(n=$ 52), 14 participants $(26.9 \%)$ showed reliable improvement, 37 (71.2\%) showed no reliable change, and one (1.9\%) showed reliable deterioration (see Table 3 ). There was a trend for males to have better outcomes on avatar-based counselling than females $(t=1.78, p=.08)$. Post hoc subgroup analysis indicated that, while the male subsample showed significant, moderate-to-large reductions in psychological distress using avatar-based counselling (mean reduction from 13.1 to 8.4 on the YP-CORE, $t=3.58, p=.002$, Cohen's $d=.61$ ), females showed small-to-moderate reductions with only a trend towards significance (mean reduction from 18.6 to 15.5 on the YP-CORE, $t=1.82, p=.08$, Cohen's $d=0.31$ ). 
There was a trend towards significant differences in outcomes across schools $(F=2.0$, $p=.07)$. Outcomes ranged from a mean improvement of 11.6 points on the YP-CORE at one site $(n=4)$ to a mean deterioration of 1.2 points on the YP-CORE at another site $(n=5)$. There was a trend towards an association between clients' outcomes and the amount of time that their counsellors had spent logged in to ProReal for training or for practice $\left(\chi^{2}=3.6, p=\right.$ .06). Clients' whose counsellors had spent more time logged into ProReal for training or practice tended to show greater reductions in psychological distress.

Improvements on the YP-CORE were not significantly related to clients' ages, their school years, their disability status, the number of sessions they attended, or the average amount of time that they used ProReal during the sessions.

\section{Psychological difficulties (SDQ)}

Participation in the avatar-based therapy was associated with significant reductions in total psychological difficulties on the SDQ $(t=2.7, p=.01)$. From pre- to post-therapy, the average score on the SDQ-TD for the total sample dropped by 1.8 points, giving a small to medium effect size of 0.28 . For this sample, significant improvements were also found on the SDQ Conduct Problems subscale $(t=2.5, p=.02, d=0.29)$, and there was a trend towards reductions in emotional symptoms from pre- to post-therapy $(t=2.7, p=.10, d=$ 0.19). However, improvements were significant for new clients only.

As with the YP-CORE, improvements on the SDQ were significant only for males. For the subsample of 21 boys, there were significant reductions on total difficulties (pretherapy mean $=14.3$, post-therapy mean $=12.0, t=2.7, p=.02, d=0.38)$, conduct problems (pre-therapy mean $=2.9$, post-therapy mean $=2.2, t=2.7, p=.02, d=0.38$ ), and a trend towards reductions in hyperactivity (pre-therapy mean $=5.5$, post-therapy mean $=5.0, t=$ $1.9, p=.08, d=0.20)$. By contrast, for females, there were no significant differences in SDQ scores between pre and post-therapy (all $p s>.24)$. 
As with the YP-CORE, there was a trend towards significant differences in SDQ-TD scores across schools $(F=2.1, p=.08)$. In addition, clients' outcomes on the SDQ-TD were significantly associated with the amount of time that their counsellors had spent logged in to ProReal for training or for practice $\left(\chi^{2}=13.1, p<.001\right)$. Clients whose counsellors had spent more time logged into ProReal for training or practice were more likely to show reductions in total psychological difficulties.

\section{Symptoms of depression and anxiety (RCADS)}

Participation in avatar-based counselling was not associated with significant reductions in symptoms of depression or anxiety $(p s>.25)$. However, there was a trend towards pre-existing clients showing a significant increase in symptoms of depression from pre- to post-therapy (pre-therapy mean $=19.7$, post-therapy mean $=21.2 ; t=-2.2, p=.05, d$ $=-0.35)$.

For males, there were significant reductions in symptoms of depression $(t=2.2, p=$ $.04, d=0.30)$ and a trend towards significant reductions in symptoms of anxiety $(t=1.9, p=$ $.07, d=0.32$ ). By contrast, for females, changes in symptoms of depression, anxiety and overall symptoms were all non-significant $(p s>.5)$.

Depression and GAD outcomes on the RCADS did not vary significantly across schools $(p \mathrm{~s}>.15)$. However, clients' outcomes on anxiety $\left(\chi^{2}=8.0, p=.004\right)$, and depression $\left(\chi^{2}=4.4, p=.04\right)$, were significantly associated with the amount of time that their counsellors had spent logged into ProReal for training or for practice. Clients whose counsellors had spent more time logged into ProReal for training or practice were more likely to show reductions in anxiety and depression.

\section{Self-reassurance (FSCRS)}

Engagement in avatar-based counselling was associated with a significant increase in levels of self-reassurance $(t=2.2, p=.03, d=0.26)$. In addition, post hoc sub-group analysis 
found that these changes were significant for males only (pre-therapy mean $=19.7$, posttherapy mean $=23.2 ; t=3.5, p=.002, d=0.52$ ).

\section{Experience of services (ESQ)}

All participants said that it was either 'certainly' or 'partly' true that the help they had received was good, with $90 \%$ giving the former rating. A summed score on the ESQ Satisfaction with Care dimension could be calculated for 38 participants. The mean score was $16.4(S D=2.1)$. Scores ranged from 10 to 18 with a median score of $17(44.7 \%$ of all respondents).

Males expressed significantly greater satisfaction with the avatar-based counselling intervention than females, with a large difference in ratings between the two groups (male mean $=17.1$, female mean $=15.6, t=2.2, p=.04, d=0.71)$. There were also significant differences in levels of satisfaction with care across schools $(F=3.2, p=.01)$. In addition, clients' satisfaction with care was significantly associated with the amount of time that their counsellors had spent logged in to ProReal for training or for practice $\left(\chi^{2}=9.7, p=.002\right)$. Clients' whose counsellors had spent more time logged into ProReal for training or practice were more likely to show greater satisfaction with the avatar-based counselling.

\section{Discussion}

In summary, participation in avatar-based counselling was associated with significant, small-to-medium reductions in psychological distress, psychological difficulties and conduct problems; and a significant, small increase in self-compassion. However, subgroup analysis indicated that these improvements were significant only for male clients, and for those starting new episodes of therapy. Male clients were also more satisfied with their experience of avatar-based counselling. There was a trend towards differences in outcomes across schools. Outcomes were greater where counsellors had spent more time in training, and using, the ProReal software. 
The intervention--as well as the broader research protocol--was feasible to implement and appeared acceptable to clients. As well as relatively low non-engagement and dropout rates $(<20 \%)$, no major ethical or procedural problems emerged. Data suggests that the ProReal software was used for about $50 \%$ of session time, indicating that clients and therapists were generally content to use it. In addition, clients' levels of satisfaction with the avatar-based counselling were acceptable and consistent with other counselling interventions for young people (e.g., Cooper, 2009).

Across outcome indicators, we found that improvements were significant for males only. This is consistent with findings from a large scale trial of an online, self-directed CBT program for students (MoodGYM, Calear et al., 2009). It is not clear why this is the case. However, given our data on differential dropout rates, and also qualitative findings from this study (van Rijn, Cooper, \& Chryssafidou, 2018), it seems possible that males may have found the digital format more familiar or comfortable than females, and therefore engaged more fully with the intervention. This finding may also reflect gender differences in therapy preferences, with female clients valuing more the interpersonal relation and the expression of feelings, while, when dealing with negative emotions, male clients may tend to prefer a more logical, systematic, and structured approach (Garber, 2006). This is a principal area for further research, as the identification of a 'male-friendly' form of therapeutic intervention could be of particular value in addressing the challenge of fewer boys accessing school-based counselling services (Cooper, 2009).

The finding that pre-existing clients did not show significant improvement in avatarbased counselling is relatively unsurprising, given the 'negatively accelerating curve' pattern of change that is typically found in counselling and psychotherapy research (Kopta et al., 1994). This indicates that, as clients have more and more sessions, the added benefit of each session diminishes. 
This pilot evaluation has a number of limitations. Data on feasibility and acceptability were mainly drawn from clients and their engagement with the intervention, and additional data from school, parent and therapist perspectives would have provided a more comprehensive analysis. In terms of outcomes, the sample size was relatively small, and this means that the findings must be treated with caution, as Type II errors may have occurred. Conversely, however, our use of a relatively liberal $p$-value, as well as the conducting of multiple tests, means that we may also be vulnerable to Type I errors: identifying differences as significant when they are, in fact, not. The use of data from pre-existing clients is also problematic, as significant therapeutic changes may already have happened, prior to the start of the avatar-based counselling intervention. All counsellors were female, and although this is not uncommon for the school counselling field it may have influenced the findings in some way. There was no control group for comparisons of outcomes, or data at follow-up time periods. Our extraction of subscales on the RCADS and the FSCRS from the context of the full measure also makes this data less reliable. Another limitation was that there may have been variability in how the intervention was implemented in different schools, and this was supported by evidence suggesting significant differences in outcomes across sites. In terms of predicting outcomes, a final important limitation is that we were testing the effects of an intervention at first implementation, rather than as delivered by seasoned therapists. This is confirmed by the significant relationship between time spent on training and practice in the intervention and outcomes.

Further research on the feasibility and acceptability of this intervention would benefit from formally assessing therapists' confidence in using this technology. An evaluation of the outcomes of avatar-based counselling, as delivered by counsellors who have gained experience and confidence in this work, would also provide a more accurate assessment of its potential results in general counselling. Randomised controlled trials would allow for a 
comparison of the effectiveness of this approach against usual pastoral care, or against an alternate counselling or digital intervention. It may also be useful to trial the effects of counselling with ProReal (as an adjunctive tool) against counselling without ProReal. Such a study, however, would need to specify the minimum amount of ProReal to be used in the former condition.

In terms of implications for practice, the findings from this study suggest that counsellors and schools should consider the use of ProReal as an adjunct to school-based humanistic counselling, particularly for young males. Counsellors using this software, however, will need to ensure that they have had sufficient levels of training and practice. In addition, the outcomes of avatar-based counselling should be evaluated on an ongoing and regular basis (for instance, using weekly YP-CORE forms), to deepen an understanding of the outcomes of this approach.

In conclusion, avatar-based counselling using ProReal is feasible to implement, and evaluate, within a school setting. It is associated with small to moderate reductions in psychological problems. There is preliminary evidence, however, of gender differences: with the intervention associated with significant improvements for males only. Although we found no evidence of superiority in the efficacy of avatar-based counselling over standard school-based humanistic counselling, or other forms of digital intervention, our research shows that the intervention — and its outcomes—-were at a generally acceptable level. This forms a solid basis from which to further develop this novel intervention: establishing procedures, strategies and guidelines that can optimise its efficacy for young clients.

\section{References}

Anthony, K., \& Nagel, D. (2014). Avatars - opening the virtual doors of therapy. Psychotherapy 2.0: Where Psychotherapy and Technology Meet, 133-145 
Baião, R., Gilbert, P., McEwan, K., \& Carvalho, S. (2015). Forms of Self-

Criticising/Attacking \& Self-Reassuring Scale: Psychometric properties and normative study. Psychology and Psychotherapy: Theory, Research and Practice, 88(4), 438-452. doi: 10.1111/papt.12049

Barak, A. (2007). Phantom emotions: Psychological determinants of emotional experiences on the Internet. Oxford handbook of Internet psychology, 303-329.

Brown, A., Ford, T., Deighton, J., \& Wolpert, M. (2014). Satisfaction in Child and Adolescent Mental Health Services: Translating Users' Feedback into Measurement. Administration and Policy in Mental Health and Mental Health Services Research, 41(4), 434-446. doi: 10.1007/s10488-012-0433-9

Calear, A. L., Christensen, H., Mackinnon, A., Griffiths, K. M., \& O’Kearney, R. (2009). The YouthMood Project: a cluster randomized controlled trial of an online cognitive behavioral program with adolescents. Journal of Consulting and Clinical Psychology, 77(6), 1021.

Castilho, P., Pinto-Gouveia, J., \& Duarte, J. (2015). Exploring Self-criticism: Confirmatory Factor Analysis of the FSCRS in Clinical and Nonclinical Samples. Clinical psychology \& psychotherapy, 22(2), 153-164.

Chorpita, B. F., Moffitt, C. E., \& Gray, J. (2005). Psychometric properties of the Revised Child Anxiety and Depression Scale in a clinical sample. Behaviour research and therapy, 43(3), 309-322.

Clough, B. A., \& Casey, L. M. (2011). Technological adjuncts to increase adherence to therapy: a review. Clinical Psychology Review, 31(5), 697-710

Cooper, M. (2009). Counselling in UK secondary schools: A comprehensive review of audit and evaluation studies Counselling and Psychotherapy Research, 9(3), 137-150. doi: $10.1080 / 14733140903079258$ 
Cooper, M. (2013). School-based counselling in UK secondary schools: A review and critical evaluation. Lutterworth: BACP/Counselling MindEd.

Cooper, M., Rowland, N., McArthur, K., Pattison, S., Cromarty, K., \& Richards, K. (2010). Randomised controlled trial of school-based humanistic counselling for emotional distress in young people: Feasibility study and preliminary indications of efficacy. Child and Adolescent Psychiatry and Mental Health, 4(1), 1-12. doi: 10.1186/17532000-4-12.

Cooper, M., Chryssafidou, E., \& van Rijn, B. (2016). ProReal avatar-based counselling with young people. Evaluation report.

Craig, T. K., Rus-Calafell, M., Ward, T., Leff, J. P., Huckvale, M., Howarth, E., ... \& Garety, P. A. (2018). AVATAR therapy for auditory verbal hallucinations in people with psychosis: a single-blind, randomised controlled trial. The Lancet Psychiatry, 5(1), $31-40$.

Cuijpers, P., van Straten, A., Andersson, G., \& van Oppen, P. (2008). Psychotherapy for depression in adults: a meta-analysis of comparative outcome studies. Journal of Consulting and Clinical Psychology, 76(6), 902-922.

Eichenberg, C., \& Wolters, C. (2012). Virtual Realities in the treatment of mental disorders: A Review of the current state of research: INTECH Open Access Publisher.

Falconer, C. J., Cutting, P., Davies, E. B., Hollis, C., Stallard, P., \& Moran, P. (2017). Adjunctive avatar therapy for mentalization-based treatment of borderline personality disorder: a mixed-methods feasibility study. Evidence-based mental health, ebmental2017.

Fleming, T., Dixon, R., Frampton, C., \& Merry, S. (2012). A pragmatic randomized controlled trial of computerized CBT (SPARX) for symptoms of depression among 
adolescents excluded from mainstream education. Behavioural and cognitive psychotherapy, 40(5), 529-541.

Fleming, T. M., Cheek, C., Merry, S. N., Thabrew, H., Bridgman, H., Stasiak, K., . . Hetrick, S. (2015). Serious games for the treatment or prevention of depression: a systematic review. Revista de Psicopatología y Psicología Clínica, 19 (16).

Gaggioli, A., Mantovani, F., Castelnuovo, G., Wiederhold, B., \& Riva, G. (2003). Avatars in clinical psychology: a framework for the clinical use of virtual humans. Cyberpsychology \& behavior, 6(2), 117-125.

Garber, J. (2006). Depression in children and adolescents: linking risk research and prevention. American Journal of Preventive Medicine, 31(6), 104-125.

Gilbert, P., Clarke, M., Hempel, S., Miles, J., \& Irons, C. (2004). Criticizing and reassuring oneself: An exploration of forms, styles and reasons in female students. British Journal of Clinical Psychology, 43(1), 31-50.

Goodman, R., Meltzer, H., \& Bailey, V. (1998). The strengths and difficulties questionnaire: A pilot study on the validity of the self-report version. European Child and Adolescent Psychiatry, 7, 125-130.

Hagquist, C. (2007). The psychometric properties of the self-reported SDQ - An analysis of Swedish data based on the Rasch model. Personality and Individual Differences, 43(5), 1289-1301.

Hoch, D. B., Watson, A. J., Linton, D. A., Bello, H. E., Senelly, M., Milik, M. T., ... \& Kvedar, J. C. (2012). The feasibility and impact of delivering a mind-body intervention in a virtual world. Plos one, 7(3), e33843.

Hill, A., Roth, A., \& Cooper, M. (2013). The competences required to deliver effective humanistic counselling for young people. Lutterworth: BACP.Joinson, A.N. (2001). Self- disclosure in computer- mediated communication: The role of self- awareness 
and visual anonymity. European journal of social psychology, 31(2), 177-

192.Kendzor, D. E., \& Hébert, E. T. (2017). The best of both worlds: Avatar-assisted therapy offers the benefits of therapist-assisted and Internet-based interventions. The American Journal of Drug and Alcohol Abuse, 1-3.

Knoetze, J. (2013). Sandworlds, storymaking, and letter writing: the Therapeutic Sandstory Method. South African Journal of Psychology, 43(4), 459-469.

Kopta, S. M., Howard, K. I., Lowry, J. L., \& Beutler, L. E. (1994). Patterns of Symptomatic Recovery in Psychotherapy. Journal of Consulting and Clinical Psychology, 62(5), 1009-1016.

Meyerbröker, K., \& Emmelkamp, P. M. (2010). Virtual reality exposure therapy in anxiety disorders: a systematic review of process-and-outcome studies. Depression and Anxiety, 27(10), 933-944.

Morina, N., Ijntema, H., Meyerbröker, K., \& Emmelkamp, P. M. (2015). Can virtual reality exposure therapy gains be generalized to real-life? A meta-analysis of studies applying behavioral assessments. Behaviour Research and Therapy, 74, 18-24.

Musiat, P., Goldstone, P., \& Tarrier, N. (2014). Understanding the acceptability of e-mental health-attitudes and expectations towards computerised self-help treatments for mental health problems. BMC psychiatry, 14(1), 109.

National Collaborating Centre for Mental Health. (2014). Full report: E-therapies systematic review for children and young people with mental health problems.

Parsons, T. D. (2015). Virtual reality exposure therapy for anxiety and specific phobias. In Encyclopedia of Information Science and Technology, Third Edition (pp. 6475-6483). IGI Global.

ProReal Ltd. (2015). Avatar-based counselling: A manual for practitioners. London: ProReal Ltd. 
Provoost, S., Lau, H. M., Ruwaard, J., \& Riper, H. (2017). Embodied Conversational Agents in Clinical Psychology: A Scoping Review. Journal of medical Internet research, 19(5).

Przeworski, A., \& Newman, M. G. (2006). Efficacy and utility of computer-assisted cognitive behavioural therapy for anxiety disorders. Clinical Psychologist, 10(2), 4353.

Rehm, I. C., Foenander, E., Wallace, K., Abbott, J.-A. M., Kyrios, M., \& Thomas, N. (2016). What Role Can Avatars Play in e-Mental Health Interventions? Exploring New Models of Richardient-Therapist Interaction. Frontiers in Psychiatry, 7, 186.

Stiles, W. B., Barkham, M., Twigg, E, Mellor-Clark, J., \& Cooper, M. (2006). Effectiveness of cognitive-behavioural, person-centred and psychodynamic therapies as practised in UK National Health Service settings. Psychological Medicine, 36(4), 555-566.

Suler, John. (2004). The online disinhibition effect. Cyberpsychology \& Behavior, 7(3), 321326.

Swan, K. L., \& Schottelkorb, A. A. (2013). Interpreting children's dreams through humanistic sandtray therapy. International Journal of Play Therapy, 22(3), 119.

Twigg, E., Barkham, M., Bewick, B. M., Mulhern, B., Connell, J., \& Cooper, M. (2009). The Young Person's CORE: Development of a brief outcome measure for young people. Counselling and Psychotherapy Research, 9(3), 160-168. doi: $10.1080 / 14733140902979722$

Twigg, E., Cooper, M., Evans, C., Freire, E., Mellor-Clark, J., McInnes, B., \& Barkham, M. (2016). Acceptability, reliability, referential distributions and sensitivity to change in the Young Person's Clinical Outcomes in Routine Evaluation (YP-CORE) outcome measure: replication and refinement. Child and Adolescent Mental Health, 21(2), 115123. doi: $10.1111 /$ camh. 12128 
van Rijn, B., Cooper, M., Jackson, A., \& Wild, C. (2015). Avatar-based therapy within prison settings: Pilot evaluation. British Journal of Guidance \& Counselling, 45(3), 268-283. doi: 10.1080/03069885.2015.1068273

van Rijn, B., Cooper, M., \& Chryssafidou, E. (2018). Avatar-based counselling for young people within school counselling. Qualitative analysis of client experience. Counselling and Psychotherapy Research, 18(1), 59-70. doi:10.1002/capr.12155

Witt, K. J., Oliver, M., \& McNichols, C. (2016). Counseling via avatar: professional practice in virtual worlds. International Journal for the Advancement of Counselling, 38(3), 218-236

Yuen, E. K., Herbert, J. D., Forman, E. M., Goetter, E. M., Comer, R., \& Bradley, J.-C. (2013). Treatment of social anxiety disorder using online virtual environments in second life. Behavior Therapy, 44(1), 51-61. 
Table 1. Participant characteristics

\begin{tabular}{crr}
\hline & $\begin{array}{r}\text { Pre-post data } \\
(\boldsymbol{n}=\mathbf{4 0})(\%)\end{array}$ & \multicolumn{1}{c}{$\begin{array}{c}\text { Full } \\
(\boldsymbol{n}=\mathbf{5 4})(\%)\end{array}$} \\
\hline Total sessions attended & & \\
$\mathbf{0}$ & $0(0.0 \%)$ & $2(3.7 \%)$ \\
$\mathbf{1}$ & $0(0.0 \%)$ & $3(5.6 \%)$ \\
$\mathbf{2}$ & $2(5.0 \%)$ & $3(5.6 \%)$ \\
$\mathbf{3}$ & $5(12.5 \%)$ & $11(20.4 \%)$ \\
$\mathbf{4}$ & $8(20.0 \%)$ & $8(14.8 \%)$ \\
$\mathbf{5}$ & $8(20.0 \%)$ & $9(16.7 \%)$ \\
$\mathbf{6}$ & $6(15.0 \%)$ & $6(11.1 \%)$ \\
$\mathbf{7}$ & $5(12.5 \%)$ & $7(9.3 \%)$ \\
$\mathbf{8}$ & $1(2.5 \%)$ & $2(3.7 \%)$ \\
$\mathbf{9}$ & $2(5.0 \%)$ & $2(3.7 \%)$ \\
$\mathbf{1 0}$ & $3(7.5 \%)$ & $3(5.6 \%)$ \\
\hline $\mathbf{1 1}$ & & \\
$\mathbf{1 2}$ & $0(0.0 \%)$ & $1(1.9 \%)$ \\
$\mathbf{1 3}$ & $10(25.0 \%)$ & $13(24.1 \%)$ \\
$\mathbf{1 4}$ & $7(17.5 \%)$ & $8(14.8 \%)$ \\
$\mathbf{1 5}$ & $7(17.5 \%)$ & $8(14.8 \%)$ \\
$\mathbf{1 6}$ & $7(17.5 \%)$ & $10(18.5 \%)$ \\
$\mathbf{1 7}$ & $3(7.5 \%)$ & $5(9.3 \%)$ \\
$\mathbf{1 8}$ & $5(12.5 \%)$ & $8(14.8 \%)$ \\
Gender & $1(2.5 \%)$ & $1(1.9 \%)$ \\
Female & & \\
Male & $19(47.5 \%)$ & $31(57.4 \%)$ \\
Ethnicity & $21(52.5 \%)$ & $23(42.6 \%)$ \\
British white & $7(17.5 \%)$ & $8(14.8 \%)$ \\
British other & $20(50.0 \%)$ & $26(48.1 \%)$ \\
Mixed & $4(10.0 \%)$ & $7(13.0 \%)$ \\
Asian & $2(5.0 \%)$ & $3(5.6 \%)$ \\
African & $2(5.0 \%)$ & $3(5.6 \%)$ \\
Irish & $1(2.5 \%)$ & $1(1.9 \%)$ \\
Caribbean & $3(7.5 \%)$ & $4(7.4 \%)$ \\
Black other & $1(2.5 \%)$ & $2(3.7 \%)$ \\
Disability & $3(7.5 \%)$ & $4(7.4 \%)$ \\
Yes & $37(92.5 \%)$ & $50(92.6 \%)$ \\
No & & \\
Client status & $11(27.5 \%)$ & $11(20.4 \%)$ \\
Pre-existing & $29(72.5 \%)$ & $43(79.6 \%)$ \\
New & & $28(51.9 \%)$ \\
Clinical & $(45.0 \%)$ & $26(48.1 \%)$ \\
\hline
\end{tabular}


Table 2. Change from Pre- to Post-Intervention (All participants)

\begin{tabular}{llrrrrr}
\hline Measure & $\boldsymbol{n}$ & $\begin{array}{l}\text { Pre- Mean } \\
(\text { SD })\end{array}$ & $\begin{array}{l}\text { Post- Mean } \\
(\text { SD })\end{array}$ & $\boldsymbol{t}$ & $\boldsymbol{p}$ & $\boldsymbol{d}$ \\
\hline YP-CORE & 52 & $16.2(9.3)$ & $12.4(9.6)$ & 3.4 & $.001^{* *}$ & 0.41 \\
SDQ-TD & 40 & $15.4(6.4)$ & $13.6(6.1)$ & 2.7 & $.01^{* *}$ & 0.28 \\
SDQ-ES & 40 & $4.8(2.6)$ & $4.3(2.7)$ & 1.7 & .10 & 0.19 \\
SDQ-CP & 40 & $2.7(1.9)$ & $2.2(1.6)$ & 2.5 & $.02^{*}$ & 0.26 \\
SDQ-HA & 40 & $5.2(2.6)$ & $4.9(2.4)$ & 1.6 & .12 & 0.12 \\
SDQ-PP & 40 & $2.7(2.0)$ & $2.4(1.8)$ & 1.3 & .19 & 0.15 \\
SDQ-PS & 40 & $7.7(1.7)$ & $8.0(1.5)$ & 1.4 & .17 & 0.18 \\
RCADS Depression & 40 & $19.6(5.8)$ & $19.4(7.1)$ & 0.2 & .86 & 0.03 \\
RCADS GAD & 40 & $12.7(4.0)$ & $11.8(3.9)$ & 1.5 & .14 & 0.23 \\
FSCRS Reassured Self & 41 & $18.4(7.5)$ & $20.3(8.2)$ & 2.2 & $.03^{*}$ & 0.25 \\
\hline
\end{tabular}
Note. YP-CORE $=$ Young Person's CORE, SDQ-TD $=$ Strengths and Difficulties Questionnaire Total

Difficulties, SDQ-ES = SDQ Emotional Symptoms, SDQ-CP = SDQ Conduct Problems, SDQ-HA = SDQ

Hyperactivity, SDQ-PP = SDQ Peer Problems, SDQ-PS = SDQ Prosocial, RCADS Depression = Revised Child

Anxiety and Depression Scale Major Depressive Disorder, RCADS GAD = Revised Child Anxiety and

Depression Scale Generalised Anxiety Disorder, FSCRS Reassured Self = Forms of Self-Criticising/Attacking and Self-Reassuring Scale's Reassured Self subscale; $d=$ effect size expressed as Cohen's $d$. Higher scores indicate greater distress excepting SDQ-PS and Self-Reassurance Scale. 
Table 3: Percentage of young people meeting criteria for reliable and clinically significant change on YP-CORE

\begin{tabular}{|l|c|c|}
\hline Reliable and clinically significant change on YP-CORE & \multicolumn{2}{|c|}{ \% } \\
\hline Reliable and clinically significant improvement & 3 & 5.8 \\
\hline Reliable improvement only (stayed clinical) & 3 & 5.8 \\
\hline Reliable improvement only (stayed non-clinical) & 14 & 26.9 \\
\hline No reliable change (stayed clinical) & 1 & 3.9 \\
\hline No reliable change but moved from clinical to non-clinical & 2 & 38.5 \\
\hline No reliable change but moved from non-clinical to clinical & 20 & 0 \\
\hline No reliable change (stayed non-clinical) & & \\
\hline Reliable deterioration (stayed clinical) & & 0 \\
\hline Reliable deterioration (stayed non-clinical) & 0 & 1.9 \\
\hline Reliable and clinically significant deterioration & 1 & 0 \\
\hline Reliable deterioration (stayed non-clinical) & & \\
\hline
\end{tabular}




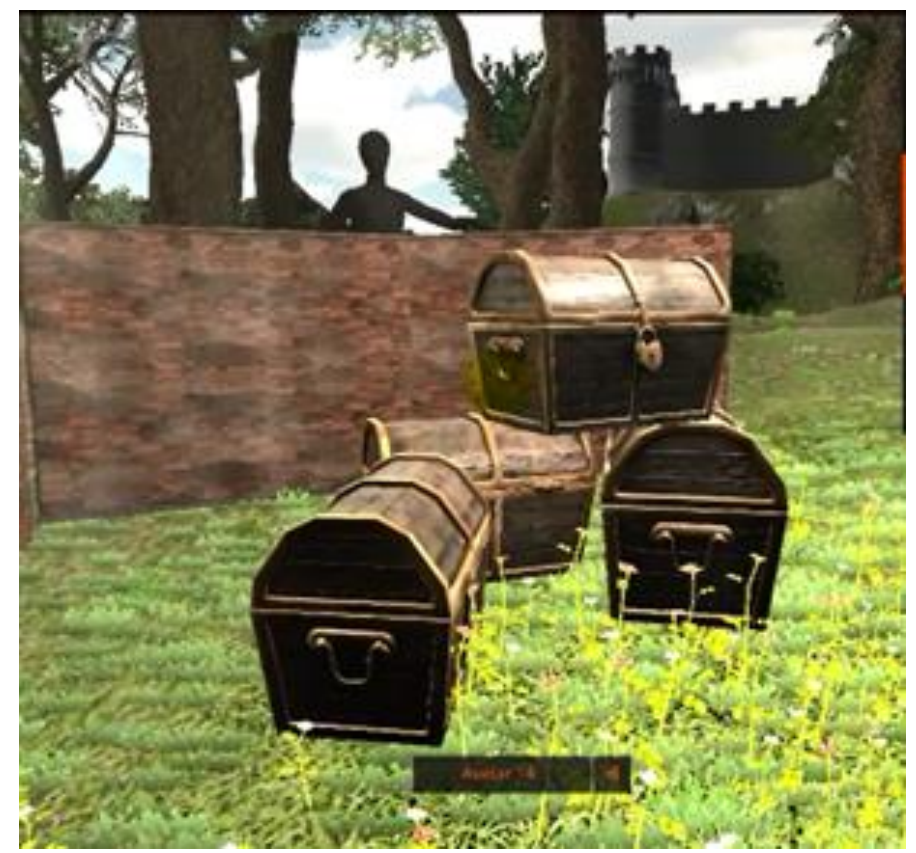

Figure 1. Richard represented his desire to be normal with large treasure chests 


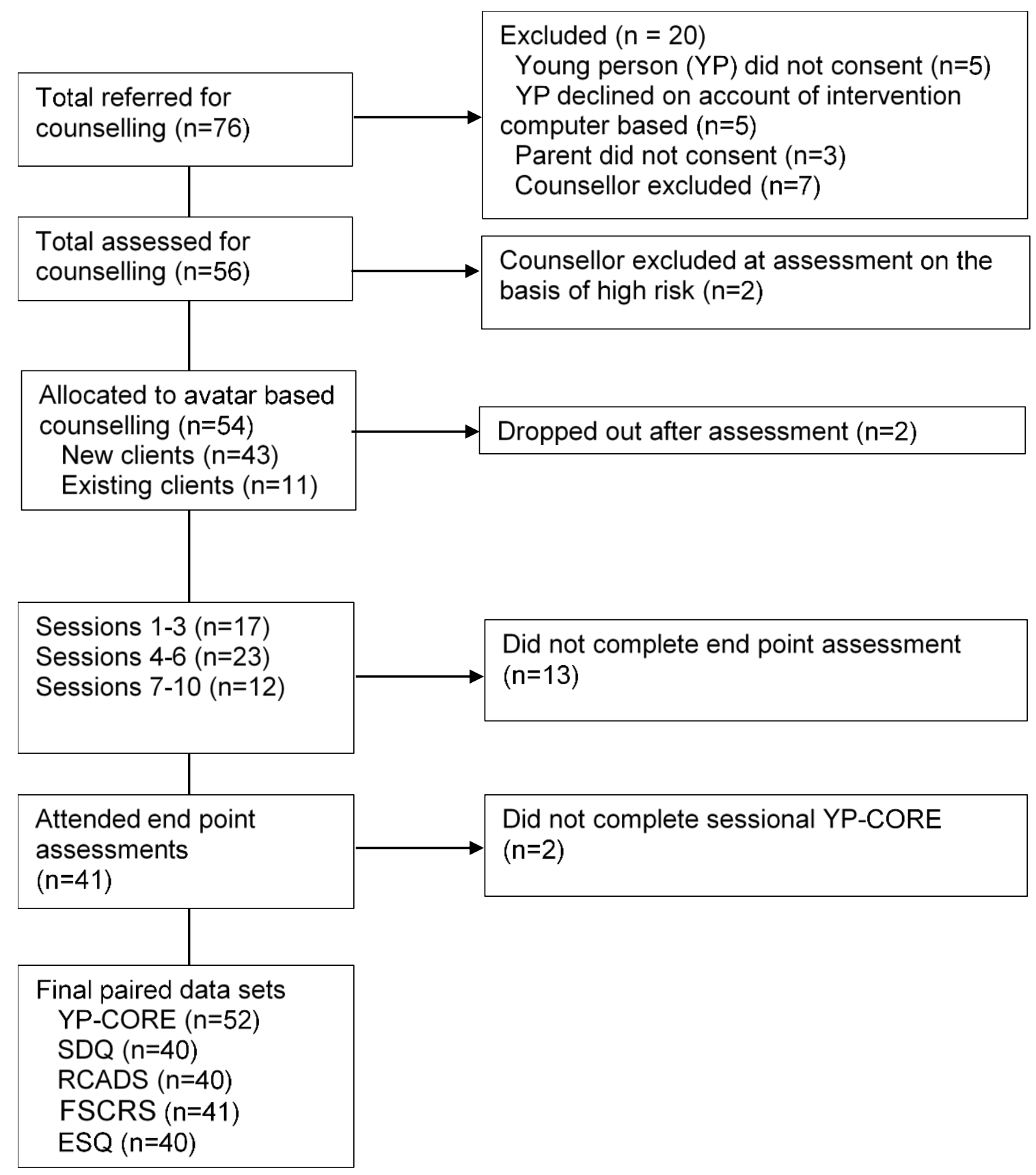

Figure 2. Participant flow chart 